\title{
Synthesis and antibiofilm activity of substituted
}

\section{1-deazapyrimido[1,2,3-cd]purinium salts}

\author{
Liubov Muzychka $^{1}$, Oksana Muzychka ${ }^{1}$, Oleg Smolii ${ }^{1}$, Iryna Boiko ${ }^{2}$, Nataliia Hrynchuk ${ }^{2}$
}

1. Department of Chemistry of Natural Compounds, V.P. Kukhar Institute of Bioorganic Chemistry and Petrochemistry of the NAS of Ukraine, UKRAINE, Kyiv, 1, Murmanska st., E-mail: lmuzychka@rambler.ru

2. Laboratory of Pharmacology of Antimicrobial Agents, SI Institute of Pharmacology and Toxicology of the NAMS of Ukraine, UKRAINE, Kyiv, 14, Anton Tsedik st., E-mail: boikoir.ol@gmail.com

\begin{abstract}
Two examples of new derivatives of 1-deazapyrimido[1,2,3-cd]purine were synthesized by intramolecular cyclization of 7-substituted 7H-pyrrolo[2,3-d]pyrimidine-6-carboxylates. The results of antimicrobial tests showed that 1-deazapyrimido[1,2,3-cd]purinium salts exhibited a pronounced effect against gram-positive and gram-negative bacteria biofilm.
\end{abstract}

Keywords - pyrrolo[2,3-d]pyrimidine, 1-deazapyrimido[1,2,3-cd]purine, quaternary salts, synthesis, antibiofilm activity.

\section{Introduction}

Quaternary ammonium salts are used in medicine and pharmacology as bactericides and antiseptics [1]. Their wide application caused the development of microbial resistance to these compounds. In recent years, much work has been devoted to the problem of preventing the formation of biofilms by bacteria [2]. Therefore, there is a need to synthesize new quaternary ammonium salts that can be used as effective disinfectants to combat both planktonic and biofilm forms of microorganisms. In addition, the antimicrobial properties of quaternary heterocyclic compounds, including 1-deazapyrimido[1,2,3-cd]purinium salts, remain poorly understood to date. Taking this into account, we synthesized a series of 1-deazapyrimido[1,2,3$c d$ ]purinium salts and evaluated their antimicrobial effect against biofilms formed by microorganisms.

\section{Results and discussion}

The synthesis of the target 5,6-dihydro-4H-1-deazapyrimido[1,2,3-cd]purine-7-ium salts $\mathbf{3}$, 6 was performed according to Scheme 1. For the synthesis of new derivatives of 1deazapyrimido[1,2,3-cd]purine, we used an approach based on the reaction of intramolecular cyclization of 7-substituted pyrrolo[2,3- $d$ ]pyrimidines [3]. The bromination of methyl 7-allyl-4(dimethylamino)-7H-pyrrolo[2,3- $d$ ]pyrimidine-6-carboxylate (1) produced 5-bromo-5,6-dihydro4H-1-deazapyrimido[1,2,3-cd]purine-7-ium bromide 3 . The interaction of pyrrolo[2,3d]pyrimidine-6-carboxylic acid 2 with iodine led to the formation of iodolactonization product 8(iodomethyl)pyrimido[5',4':4,5]pyrrolo[2,1-c][1,4]oxazine 4. Treatment of oxazine 4 with sodium methylate resulted in the formation of methyl 7-(oxiran-2-ylmethyl)-7H-pyrrolo[2,3d]pyrimidine-6-carboxylate (5). The heating of compound 4 in acetonitrile in the presence of triethylammonium chloride gave 1-deazapyrimido[1,2,3-cd]purine-7-ium salt $\mathbf{6}$ after intramolecular cyclization.

The ability of 1-deazapyrimido[1,2,3-cd] purine derivatives in subinhibitory concentrations $10 \mu \mathrm{g} / \mathrm{mL}$ and $50 \mu \mathrm{g} / \mathrm{mL}$ to prevent bacteria from biofilm formation has been tested on polystyrene plates by gentianvioletum sorption on biofilm structures, followed by its desorption with organic solvent [4]. Antibacterial activity was investigated against clinical bacterial strains of gram-positive (Staphylococcus aureus 222 (MRSA)) and gram-negative (Escherichia coli 311, Pseudomonas aeruginosa 449) bacteria used in the study. 


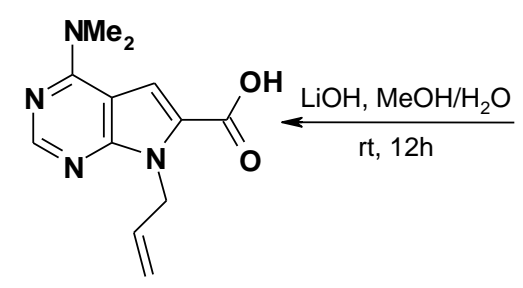

2<smiles>C=C1CC(CI)Cn2c1cc1c(N(C)C)ncnc12</smiles>

4

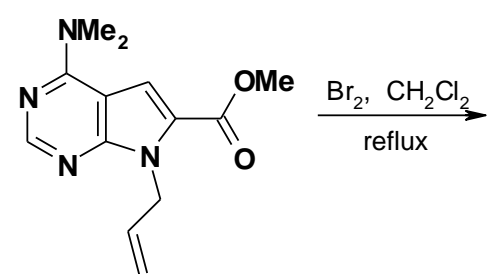

ঋ<smiles>COC(=O)c1cc2c(N)ncnc2n1CC(Br)CBr</smiles>

3

Scheme 1. Synthesis of 5,6-dihydro-4H-1-deazapyrimido[1,2,3-cd]purin-7-ium salts $\mathbf{3}, \mathbf{6}$.

Our results demonstrated that compounds $\mathbf{3}$ and $\mathbf{6}$ prevented $S$. aureus biofilm formation in a dose-dependent manner. The mass of MRSA 222 biofilm decreased by $81.2-85.0 \%$ at 50 $\mu \mathrm{g} / \mathrm{mL}$ and by $18.8-33.6 \%$ at $10 \mu \mathrm{g} / \mathrm{mL}$ when compared with untreated culture. The compound 6 inhibited E. coli and P. aeruginosa biofilms formation: the biomass decreased by $24.9 \%$ and $42.9 \%$ at concentration of $50 \mu \mathrm{g} / \mathrm{mL}$ and by $49.6 \%$ and $60.9 \%$ at concentration of $10 \mu \mathrm{g} / \mathrm{mL}$ respectively. Treatment of the gram-negative bacteria biofilm with compound $\mathbf{3}$ led to the reduction of the biomass by $31.5-38.4 \%$ (E. coli) and $12.9-18.7 \%$ (P. aeruginosa).

\section{Conclusion}

The synthesis of the two 1-deazapyrimido[1,2,3-cd]purine derivatives was developed on the basis of 7-substituted 7H-pyrrolo[2,3- $d$ ]pyrimidine-6-carboxylates. The results of antibiofilm activity showed that compounds specifically reduced the biofilm formation of S. aureus 222, E. coli 311 and P. aeruginosa 449.

\section{References}

[1] Kwaśniewska, D., Chen, Y.-L., \& Wieczorek, D. (2020). Biological activity of quaternary ammonium salts and their derivatives. Pathogens, 9(6), 459-471. https://doi:10.3390/pathogens9060459

[2] Obłąk, E., Futoma-Kołoch, B. \& Wieczyńska, A. (2021). Biological activity of quaternary ammonium salts and resistance of microorganisms to these compounds. World Journal Microbiology and Biotechnology, 37, 22. https://doi.org/10.1007/s11274-020-02978-0

[3] Muzychka, L. V., Yaremchuk, I. O., Verves, E. V., \& Smolii, O.B. (2019). Pyrrolo[2,3d]pyrimidine derivatives in the synthesis of a novel heterocyclic system $2 a, 5 a, 7-$ triazaacenaphthylene. Chemistry of Heterocyclic Compounds, 55(4-5), 397-400. https://doi:10.1007/s10593-019-02471-z

[4] O'Toole, G. A. (2011). Microtiter dish biofilm formation assay. Journal of Visualized Experiments, 47. pii: 2437. https://doi: 10.3791/2437 